\title{
The flow of power law fluids between parallel plates with shear heating
}

\author{
M. S. Tshehla, T. G. Myers \& J. P. F. Charpin \\ Department of Mathematics and Applied Mathematics, \\ University of Cape Town, South Africa
}

\begin{abstract}
A mathematical model for the flow and heat transfer between two parallel plates is studied, using the power law model. The flow due to a pressure gradient and flow due to a moving upper plate are investigated. In the derivation the Navier-Stokes and energy equations is reduced in line with the lubrication theory to provide scalar differential equations. The velocity and temperature profiles are determined analytically and the results show that the power law index $n=1$ compares favourably with Newtonian profiles. The temperature field is increasing when $n$ increases. The Brinkman number $B r$, also shows a significance increase of the temperature field when $\mathrm{Br}$ increases.

Keywords: lubrication theory, non-Newtonian flow, power law viscosity, shear heating.
\end{abstract}

\section{Introduction}

In a typical operating situation lubricants can be subjected to extreme conditions, such as high temperature, high pressure and shear rate. External heating and high shear rates can lead to high temperature being generated within a fluid. Viscosity is the most sensitive fluid property that represents a material's internal resistance to deform, see $[8,9]$. In this paper the main focus will be on the effect of viscosity variation due to the power law model see, $[3,5,6]$ for example. However for possible prediction of the results, the viscosity is considered to be constant in section 2.1 and is allowed to vary in section 2.2. Similar laws are discussed, see Andersson and Valnes [1] and Zheng and Zhang [10] in their study of boundary layer flow along lubricated surface and for squeeze flow in Lian et al [4]. Conclusion is included in section 3 . Fluids in which, viscosity is the only property to 


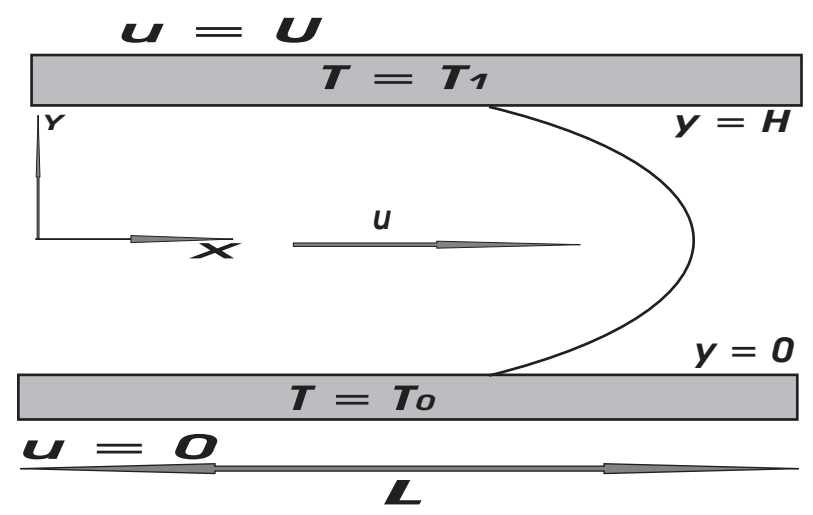

Figure 1: Schematic representation of the problem.

vary are termed generalised non-Newtonian fluids, see Schetz and Fuhs [7] and Shames [8]. Considering a basic flow configuration, where the fluid flows between parallel plates, will isolate the effect of viscosity variation. The temperature at the top and bottom plates is fixed. The lubrication theory will be exploited to reduce the governing equations to a more tractable form.

\section{Governing equations}

Two parallel plates geometrically define the problem in fig. 1. The independent variables $x$ denote the horizontal distance along the channel, $y$ the vertical distance, $H$ the distance apart and $L$ denotes typical length. The pressure and shear driven cases are combined. For the pressure driven case both plates are fixed. For the shear driven case the upper plate is moving at the speed $U$ relative to the lower plate. The upper and lower plates are maintained at $T_{1}$ and $T_{0}$ respectively. With the hypothesis established above, the appropriate equations for modelling of this problem, the continuity, Navier-Stokes and energy equations are now stated. Initially the dynamic viscosity, density, thermal conductivity and the coefficient of thermal expansion denoted by $\mu, \rho, \kappa$ and $\beta$ respectively are assumed to be constants. The Navier-Stokes equations are combined with the energy equations to solve for the velocity $u$, the pressure $p$ and the temperature $T$ of the fluid. See $[2,7,9]$.

For incompressible fluids the governing equations may be written, Continuity:

$$
\rho(\nabla \cdot \mathbf{u}),=0
$$

Navier-Stokes equation:

$$
\rho\left(\frac{\partial \mathbf{u}}{\partial t}+(\mathbf{u} \cdot \nabla) \mathbf{u}\right)=-\nabla p+\rho \mathbf{g}+\nabla^{2}(\mu \mathbf{u})
$$


Energy equation:

$$
\rho c_{p}\left(\frac{\partial T}{\partial t}+(\mathbf{u} \cdot \nabla) T\right)=\beta T\left[\frac{\partial p}{\partial t}+(\mathbf{u} \cdot \nabla) p\right]+\nabla \cdot(\kappa \nabla T)+\Phi .
$$

where,

$$
\Phi=\mu\left[2\left(\frac{\partial u}{\partial x}\right)^{2}+2\left(\frac{\partial v}{\partial y}\right)^{2}+\left(\frac{\partial u}{\partial y}+\frac{\partial v}{\partial x}\right)^{2}\right] .
$$

The components of the velocity vector $\mathbf{u}$ are denoted $(u, v)$ in the $(x, y)$ direction, $t$ denotes time. The notation is discussed in the nomenclature table 1 . Since the fluid is considered to flow in a thin layer, the governing equations can be simplified using this geometrical property. To determine the leading order terms, the governing equations will be non-dimensionalised. An asymptotic simplification known as lubrication theory may be used to simplify the governing equations. This method is valid when the film is thin and the flow regime is laminar. See [7, 8, 9]. The variables are scaled in the following manner,

$$
\begin{array}{clr}
x=L x^{\prime}, & y=H y^{\prime}, & u=U u^{\prime}, \\
v=\frac{H U}{L} v^{\prime}, & t=\frac{L}{U} t^{\prime}, & \mu=\mu_{0} \mu^{\prime}, \\
T=T_{0}+\Delta T_{0} T^{\prime}, & p=P p^{\prime}=\frac{\mu_{0} U L}{H^{2}} p^{\prime} .
\end{array}
$$

where all quantities with prime denote non-dimensional parameters. Since the film is thin the aspect ration $\epsilon=H / L \ll 1$. Using the scaled parameters eqns. (1)-(3) may now be reduced to their final form:

$$
\begin{aligned}
\frac{\partial u}{\partial x}+\frac{\partial v}{\partial y} & =0, \\
-\frac{\partial p}{\partial x}+\frac{\partial}{\partial y}\left(\mu \frac{\partial u}{\partial y}\right) & =0, \\
\frac{\partial p}{\partial y} & =0, \\
\frac{\partial^{2} T}{\partial y^{2}}+\mu B r\left(\frac{\partial u}{\partial y}\right)^{2} & =0 .
\end{aligned}
$$

where $B r=\left(\mu_{0} U^{2} / \kappa_{0} \Delta T_{0}\right), P e=\left(\rho_{0} c_{p} U L / \kappa_{0}\right)$ and $R e=\left(\rho_{0} U L / \mu_{0}\right)$ represents the Brinkman, the Peclet and Reynolds numbers respectively. Despite the fact that the Peclet number is large, the reduced Peclet number $\epsilon^{2} P e$ and the reduced Reynolds number $\epsilon^{2} R e$ are small and may be neglected in the governing equations. The Brinkman number may be close to a unity and may be retained.

Derivation of the velocity and temperature profiles may be completed after the boundary conditions associated with eqns. (4)-(7) are stated. A no slip boundary 
condition is applied at the bottom of the channel. The velocity at the top of the plate moves at a constant velocity $U$ hence the boundary conditions are listed as,

$$
\begin{array}{ll}
y=0, & u=0, \\
y=1, & u=U=1 .
\end{array}
$$

The temperature at the top and bottom of the plate are given as follows,

$$
\begin{aligned}
& y=0, \quad T=0, \\
& y=1, \quad T=1 .
\end{aligned}
$$

The final equations will be solved using the boundary conditions above.

\subsection{Newtonian model with constant viscosity}

In this case for prediction of the correct results, we start with a simple case when the viscosity is constant. In the latter stage the viscosity will be allowed to vary. Now if the viscosity is considered to be constant, the velocity is determined by integrating eqn. (5) with respect to $y$. Using the boundary conditions (8) and (9) respectively to obtain,

$$
u=\frac{1}{2}\left[\frac{\partial p}{\partial x}\left(y^{2}-y\right)\right]+U y .
$$

The first term on the right hand side is the standard parabola for the pressure driven flow. The last term is the classical straight line for the shear driven flow. The flux is determined by integrating eqn. (12) from $y=0$ to $y=1$. If the flux is constant the final integration leads to a linear pressure profile along the channel

$$
p=-3 Q\left(x-x_{0}\right)+\frac{3 U}{2}\left(x-x_{0}\right)+p_{0},
$$

where $p_{0}$ is the pressure and $x_{0}$ is the position at the inlet. The governing equation for the temperature profiles requires the velocity gradient, this gradient may be determined by differentiating eqn. (12) with respect to $y$ and combined with eqn. (7) to obtain,

$$
\frac{\partial^{2} T}{\partial y}=-\frac{B r}{4}\left[\left(\frac{\partial p}{\partial x}\right)^{2}\left(4 y^{2}-4 y+1\right)+2 U \frac{\partial p}{\partial x}(2 y-1)+U^{2}\right] .
$$

Integrating eqn. (14) twice with respect to $y$, applying the boundary conditions (10) and (11) yields,

$$
T=-\frac{B r}{4}\left[\frac{1}{12}\left(\frac{\partial p}{\partial x}\right)^{2}\left[\left(4 y^{4}-8 y^{3}+6 y^{2}-2 y\right)+\frac{U}{3} \frac{\partial p}{\partial y}\left(2 y^{3}-3 y^{2}+y\right)\right]\right.
$$




$$
\left.+\frac{U^{2}}{2}\left(y^{2}-y\right)\right]+y \text {. }
$$

The first term in the square brackets shows that the temperature is quartic in $y$ and it occurs due to the pressure gradient. The second term appears due to the combination of the pressure and the shear. The last term is the straight-line distribution. When the viscosity is not constant this simple analysis cannot be followed through. In the next section, a specific case will be studied where the flow regime obeys the power law model.

\subsection{The power law model for variable viscosity in conduits}

In the previous section the study was conducted for constant viscosity, in this section the power law model with varying viscosity will be investigated. The velocity and the temperature profiles will be derived using the power law model:

$$
\mu=m\left|\frac{\partial u}{\partial y}\right|^{n-1}
$$

where $m$ is a constant and $n$ is the power law index, $(\partial u / \partial y)$, is the shear rate. Setting $n=1$ and $m=\mu$, the Newtonian case will be retrieved. With $n \neq 1$, Eqn. (16) represent shear-thinning fluids, for $n<1$, represent pseudo-plastic fluids and $n>1$ a dilatant fluids. The absolute value sign may lead to a regularized power law model; consider the following two assumptions,

- Case (a), $\Longrightarrow,(\partial u / \partial y) \geq 0$ :

- Case (b), $\Longrightarrow,(\partial u / \partial y) \leq 0$ :

Integrating eqn. (5) with respect to $y$ yields

$$
\mu \frac{\partial u}{\partial y}=\left(G_{x} y+C_{1}\right)
$$

where $G_{x}=(\partial p / \partial y)$, Combining eqns. (16) and (17) gives,

$$
\left|\frac{\partial u}{\partial y}\right|^{n-1} \frac{\partial u}{\partial y}=\frac{\left(G_{x} y+C_{1}\right)}{m} .
$$

Using case (a) above, the positive velocity gradient is given by,

$$
\frac{\partial u}{\partial y}=\left[\frac{\left(G_{x} y+C_{1}\right)}{m}\right]^{\frac{1}{n}}
$$

Integrating eqn. (19) with respect to $y$, and applying the boundary conditions (8) and (9) gives the velocity profile as,

$$
u=\frac{n m}{(n+1) G_{x}}\left[\left(\frac{G_{x}}{m} y+\frac{C_{1}}{m}\right)^{\frac{n+1}{n}}-\left(\frac{C_{1}}{m}\right)^{\frac{n+1}{n}}\right],
$$


where $C_{1}$ will be computed from,

$$
U=\frac{n m}{(n+1) G_{x}}\left[\left(\frac{G_{x}}{m}+\frac{C_{1}}{m}\right)^{\frac{n+1}{n}}-\left(\frac{C_{1}}{m}\right)^{\frac{n+1}{n}}\right] .
$$

To determine the temperature profile, eqns. (7) and (19) are combined to give,

$$
\frac{\partial^{2} T}{\partial y^{2}}=-m B r\left[\left(\frac{G_{x} y}{m}+\frac{C_{1}}{m}\right)^{\frac{n+1}{n}}\right] .
$$

Integrating eqn. (22) twice with respect to $y$, and applying the boundary conditions (10) and (11) to eliminate the constants of integration gives the temperature profile as,

$$
\begin{aligned}
T & =-A_{1}\left[\left(\frac{G_{x} y}{m}+\frac{C_{1}}{m}\right)^{\frac{3 n+1}{n}}\right] \\
& +A_{1}\left[\left(\left(\frac{G_{x}}{m}+\frac{C_{1}}{m}\right)^{\frac{3 n+1}{n}}-\left(\frac{C_{1}}{m}\right)^{\frac{3 n+1}{n}}\right) y-\left(\left(\frac{C_{1}}{m}\right)^{\frac{3 n+1}{n}}\right)\right]+y,
\end{aligned}
$$

where $A_{1}=\frac{\left(n^{2} m^{3} B r\right)}{\left((2 n+1)(3 n+1) G_{x}^{2}\right)}$.

For the case (b) above, the same procedure may be followed to obtain the velocity and temperature profiles. The results for case (a), will be plotted and discussed quantitatively.

\subsection{Results and discussions}

Three curves representing the velocity profiles for eqn. (12) are shown in fig.2. Curve (a) represents the parabolic profile for the pressure driven flow. The maximum velocity of the flow appears at the centre of plate. Curve (b) represents a linear profile for the shear driven flow, as expected from eqn. (12) the velocity increases linearly from 0 at the lower boundary to 1 at the upper boundary. Curve (c) is the combination of both the shear and pressure driven flow. We observe a parabolic profile with its maximum velocity near the moving upper plate.

Three temperature profiles corresponding to eqn. (15) are shown in fig. 3. Curve (a) represents the shear driven case. This is a parabolic profile with its maximum temperature near the moving plate. Curve (b) represents the pressure driven case, and curve (c) represents the combination of both the pressure and shear driven cases, the parabolic type profiles with its maximum temperature towards the moving plate are shown. These results are standard for lubrication theory and may be retrieved in $[8,9]$.

Fig. 4, represent three velocity profiles for eqn. (21). Each curve corresponds to three different values of the power law index $n=0.8, n=1$ and $n=1.5$ 


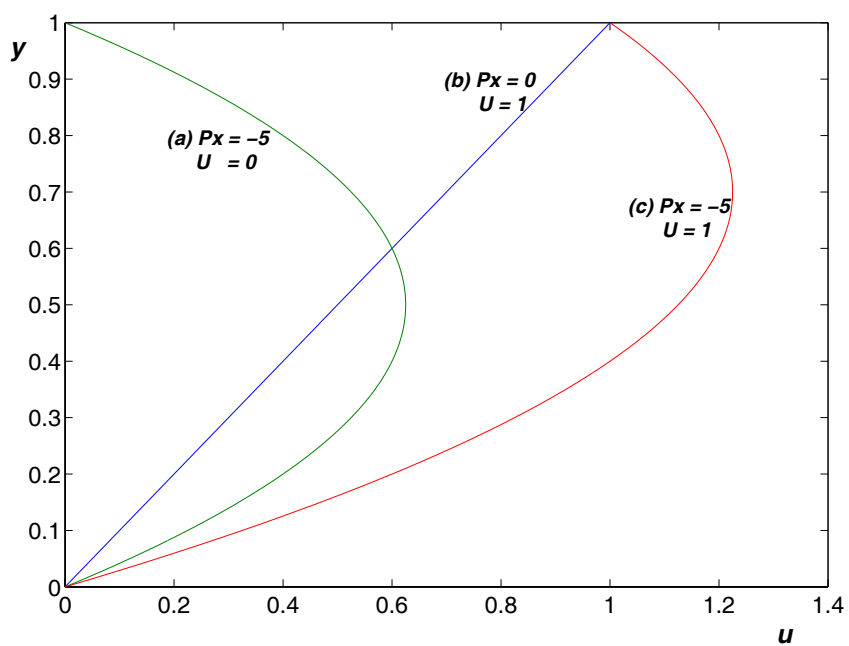

Figure 2: The velocity profile for eqn (12).

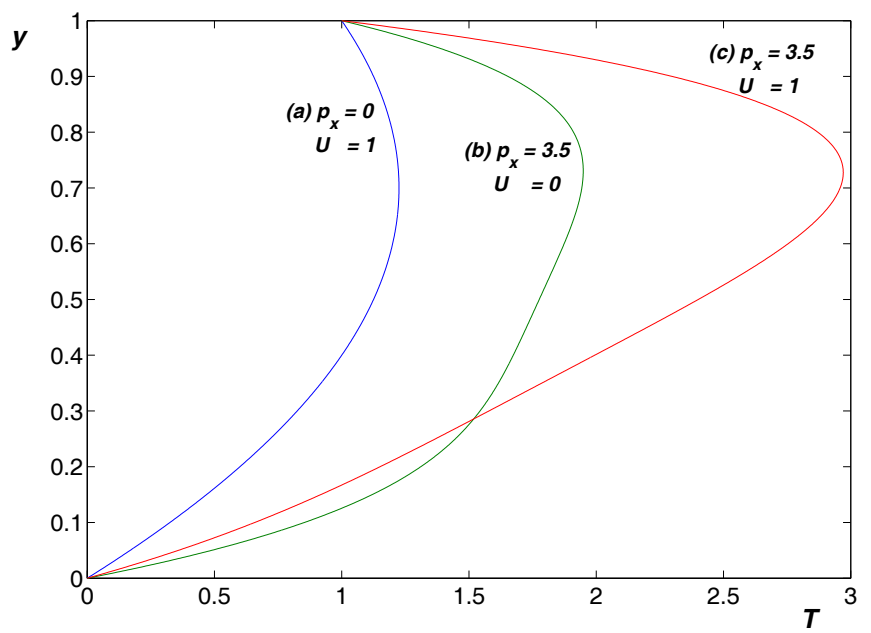

Figure 3: The temperature profile for eqn. (15).

respectively. All these curves are parabolic in shape and begin at the origin due to the boundary conditions. These curves show an increasing velocity profile with their maximum velocities towards the upper plate. Curve (b) with $n=1$ compares closely with the Newtonian case. The velocity profiles increase with an increasing power law index $n$. 


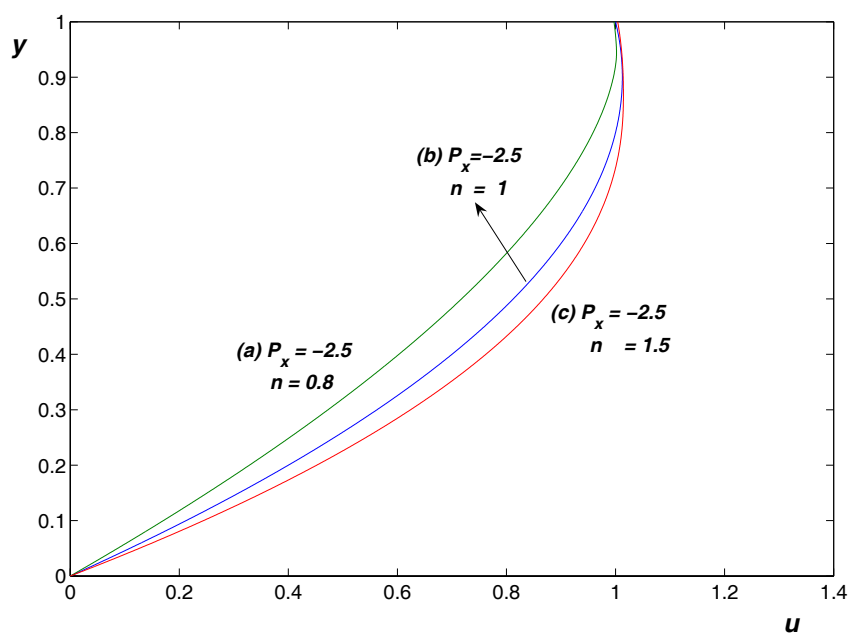

Figure 4: The velocity profile for eqn. (21).

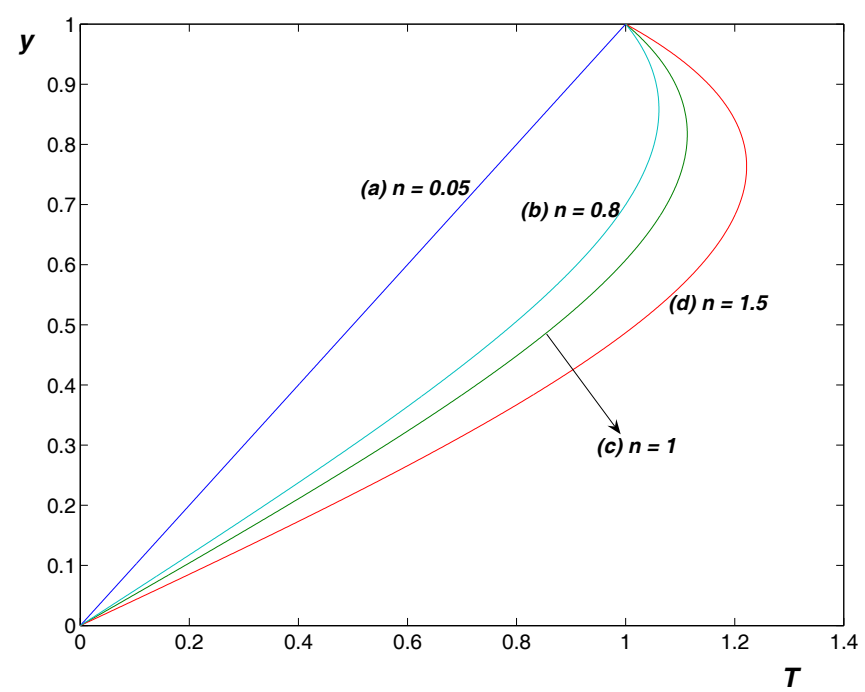

Figure 5: The temperature profile for eqn. (23).

On fig. 5, four curves representing the temperature profile for eqn. (23) are shown. Different values for $n$ namely $n=0.05, n=0.8, n=1$ and $n=1.5$ are shown respectively on the corresponding curves. A linear profile is observed in curve (a) with its maximum temperature $T=1$ at $y=1$. Three parabolic type 


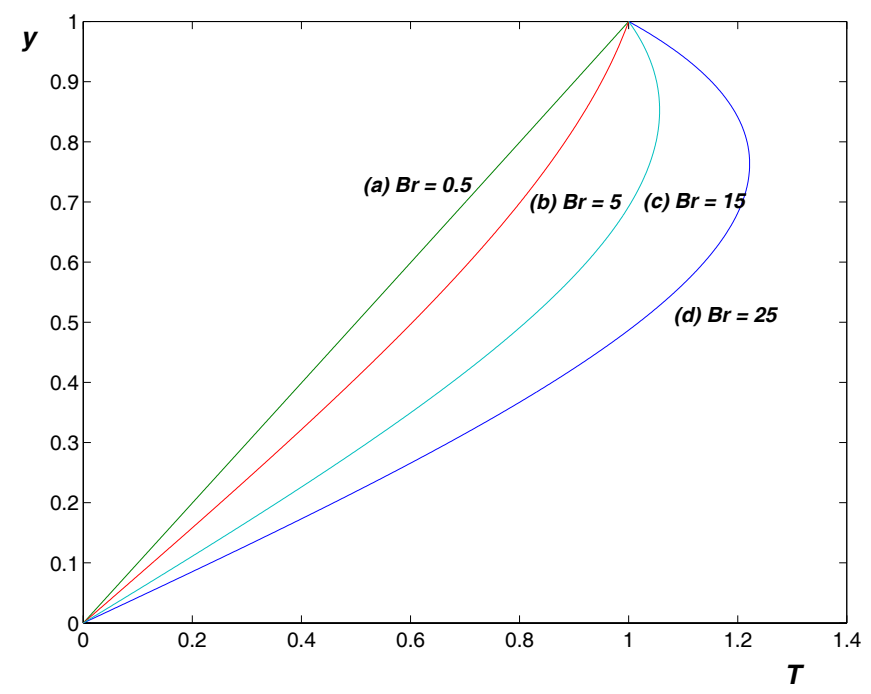

Figure 6: The temperature profile for eqn. (23) with varying $\mathrm{Br}$.

temperature profiles are observed in curves (b), (c) and (d) respectively. When $n$ increases the viscosity increases, and the temperature increases. The more viscous is the fluid the higher the temperature.

The effect of Brinkman number is investigated. Large values of $\mathrm{Br}$ means that the viscosity of the fluid is high. Fig. 6, shows four curves representing the temperature profile as shown in fig. 5, (b). Each curve corresponds to different values of $\mathrm{Br}$, namely $\mathrm{Br}=0.5, \mathrm{Br}=5, \mathrm{Br}=15$ and $\mathrm{Br}=25$ respectively. A linear profile is observed in curve (a) which is increasing across the layer to the top plate $T=1$ at $y=1$. Curve (b) also increases nonlinearly across the layer to the upper plate. Curves (c) and (d) shows parabolic profiles with their maximum temperatures $T=1.03$, at $y=0.86$ and $T=1.22$, at $y=0.78$ respectively. When $B r$ increases, a significant increase in the temperature field is observed. This shows that the temperature rise due to heat dissipation is significant when $\mathrm{Br}$ increases from $B r=0.5$ to $B r=25$.

\section{Conclusion}

In this paper the problem of applying a thin layer of a power-law fluid between parallel plates has been examined using the lubrication theory. The hydrodynamics of equivalently Newtonian model with constant viscosity was studied. The integral solutions for the velocity and temperature are presented and their flow patterns were compared. It is clear that the Newtonian results compares closely with power law model results particularly when the power law index $n=1$, see figs. 2 and 4 . 
The results shown in figs. 3 and 5, a significance increase in the power law index $n$ results in a significance increase in the both the velocity and temperature profiles. Fig. 6 clearly shows an increase in $\mathrm{Br}$ also influences the temperature. In future we hope to improve the model by allowing the temperatures at the plate to vary in the direction of the flow.

\section{Acknowledgements}

The authors acknowledge the support of this work by the National Research Foundation of South Africa, under grant number 2053289. Mr Tshehla acknowledges the department of Defence for the continuous support. Dr Charpin acknowledges the support of the Claude Leon Foundation.

\section{Nomenclature}

The following dependent variables are taken into consideration.

\begin{tabular}{|l|l|l|l|}
\hline Name & Symbol & $\begin{array}{l}\text { Typical } \\
\text { value }\end{array}$ & Unit \\
\hline$B r=\eta_{0} U^{2} /\left(k \Delta T_{m}\right)$ & Brinkman number & $0.01-0.5$ & \\
\hline$c_{p}$ & Heat capacity & 2000 & $\mathrm{~J} \cdot \mathrm{kg}^{-1} \cdot \mathrm{K}^{-1}$ \\
\hline$H$ & Channel height & $10^{-6}$ & $\mathrm{~m}$ \\
\hline$k$ & Thermal conductivity & 0.17 & $\mathrm{~W} \cdot \mathrm{m}^{-1} \cdot \mathrm{K}^{-1}$ \\
\hline$L$ & Channel length & 0.005 & $\mathrm{~m}$ \\
\hline$P$ & Pressure scale & $10^{9}$ & $\mathrm{~Pa}$ \\
\hline$p$ & Pressure & & $\mathrm{Pa}$ \\
\hline$P e=\rho c_{p} L U / k$ & Peclet number & $10^{5}$ & \\
\hline$R e=\rho U L / \eta_{0}$ & Reynolds number & $40-2000$ & \\
\hline$t$ & Time & & $\mathrm{s}$ \\
\hline$T$ & Temperature & & $\mathrm{K}$ \\
\hline$\Delta T$ & Temperature drop & 100 & $\mathrm{~K}$ \\
\hline$U$ & Velocity scale & 5 & $\mathrm{~m} / \mathrm{s}$ \\
\hline$(u, v)$ & Cartesian velocity & & $\mathrm{m} / \mathrm{s}$ \\
\hline$(x, y)$ & Cartesian coordinates & & $\mathrm{m}$ \\
\hline$\epsilon$ & Aspect ratio of the flow & $10^{-4}$ & \\
\hline$\eta$ & Dynamic viscosity & & $\mathrm{kg} \cdot \mathrm{m}^{-1} \cdot \mathrm{s}^{-1}$ \\
\hline$\eta_{0}$ & Typical dynamic viscosity & $0.01-0.5$ & $\mathrm{~kg} \cdot \mathrm{m}^{-1} \cdot \mathrm{s}^{-1}$ \\
\hline$\rho$ & Fluid density & 1000 & $\mathrm{~kg} \cdot \mathrm{m}^{-3}$ \\
\hline
\end{tabular}




\section{References}

[1] Andersson, H. I. \& Valnes, O. A., Slip-flow boundary conditions for nonNewtonian lubrication layers. Fluid Dynamic Research, 24, pp. 211-217, 1999.

[2] Constatinescu, V. N., Laminar Viscous flow, Springer-Verlag: New York, pp. 127-134, 1995.

[3] Gupta, R. C., On developing laminar no-Newtonian flow in pipes and channels. Nonlinear Analysis, 2, pp. 171-193, 2001.

[4] Lian, G. Xu, Y. Huang, W., \& Adams, M. J., On the squeeze flow of a power law between rigid spheres. Journal of non-Newtonian fluid Mechanics, 100, pp. 151-164, 2001.

[5] Rao, B. K., Heat transfer to falling power law. Heat and Fluid flow, 20, pp. 429-436, 1999.

[6] Ross, A. B. Wilson, S. K. \& Duffy, B. R., Blade coating of power-law fluid. Physics of Fluids, 11(5), pp. 958-970, 1999.

[7] Schetz, J. A. \& Fuhs, A. E., Fundamentals of fluid mechanics, John Wiley and Sons: USA, pp. 243, 1999.

[8] Shames, I. H., Mechanics of fluids, McGraw-Hill: USA, pp. 14, 1992.

[9] White, F. M., Viscous fluid flow, McGraw-Hill: USA, pp. 235-238. 1991.

[10] Zheng, L. C. \& Zhang X. X., Skin friction and heat transfer in power-law fluid laminar boundary layer along a moving surface. International Journal of Heat Mass transfer, 45, pp. 2667-2672, 2002. 